\title{
Never Give Up: To Use POCUS For Emergency Patient
}

\author{
Betul Gulalp ${ }^{1}$, Elif Karadeli ${ }^{2}$ \\ Departments of ${ }^{1}$ Emergency Medicine and ${ }^{2}$ Radiology, Adana Training and Research Center, Baskent University, Adana, Turkey.
}

\section{Corresponding Author: \\ Dr. Betul Gulalp \\ Email: docbetul@yahoo.com}

This is an Open Access article distributed under the terms of the Creative Commons Attribution License (creativecommons.org/ licenses/by/3.0).

Received Accepted

Published

February 18, 2017

May 17, 2017

June 20, 2017

\begin{abstract}
Background: Point of care ultrasonography (POCUS) is modality being used in emergency medicine for last two decades. Case Report: A 76 year old man was referred to the emergency department as flank pain and urolithiasis. However, he complained of dyspnea for a few days. At his triage examination oxygen saturation was $82 \%$ in room air. Pulmonary examination revealed bilateral rales with decreasing frequency, decreased respiratory sounds, and elongated expiration. A large-curve transducer (Philips HDI 5000 C5-2R $15 \mathrm{~Hz}$ transducer) was used to examine the apical four chamber view on the obese patient in full sitting position leaning forward which was the only one could be reached at the point of care. POCUS showed significant myocardial contractility of apex despite free wall hypokinesis of the right ventricle (RV) on visual qualitative evaluation of the apical four chamber view, confirmed as pulmonary thromboembolism of the bilateral branches of the pulmonary arteries. Conclusion: Point-of-care ultrasound can be extremely helpful tool to aid clinical assessment in emergency situations.
\end{abstract}

Keywords: Dypnea, Emergency Medicine, Oxygen, Point of Care Systems, Pulmonary Embolism.

\section{Introduction}

Point of care ultrasonography (POCUS) is the current management of emergency patient for two decades in Emergency Department (ED). McConnell sign is a possible finding of acute corpulmonary disease identified in POCUS described as a motion of apex of dilated right ventricle directed inside in systole.

\section{Case Report}

A 76 year old man was referred to the ED with flank pain and urolithiasis. However, he had dyspnea. At his triage examination his temperature was $36^{\circ} \mathrm{C}$, heart rate was 91 beats $/ \mathrm{min}$, respiratory rate was $23 / \mathrm{min}$, blood pressure was $100 / 60 \mathrm{~mm} \mathrm{Hg}$, oxygen saturation was $82 \%$ in room air, and blood glucose was $138 \mathrm{mg} / \mathrm{dL}$.

No murmurs, clicks, rubs, or gallops were detected upon cardiac auscultation. Pulmonary examination revealed bilateral rales with decreasing frequency, decreased respiratory sounds, and elongated expiration. There was no tenderness in the lower extremities, color and temperature were normal. The patient exhibited 1+ pretibial edema. The only additional significant finding of the physical examination was left costochondral angle tenderness. The patient excreted $450 \mathrm{cc}$ of urine in the urinary catheter that had been placed in the emergency department (ED).

The electrocardiogram (ECG) revealed the presence of a right bundle block. Point-of-care cardiac ultrasound was performed in the ED to assist in the differential diagnosis of this critical patient. A large-curve transducer (Philips HDI 5000 C5-2R $15 \mathrm{~Hz}$ transducer), the only one could be reached was used to examine the apical fourchamber view on the patient in full sitting position and leaning forward. This was the only device at the point of care in ED at that moment. The 
patient was obese and could not tolerate a reclined position. Visual evaluation of the apical four chamber view using POCUS showed significant myocardial contractility of apex despite free wall hypokinesis of the dilated right ventricle (RV) with a hypertrophic and hyperkinetic left ventricle on video [Fig.1]. Computed tomography (CT) angiography of the chest demonstrated pulmonary thromboembolism of the bilateral branches of the pulmonary arteries [Fig.2] with frosted glass appearance, cardiomegaly, and pleural effusion of the right side. The physical examination and vital signs, diagnosis, clinical management and hospitalization decision occurred within approximately five minutes as a result of the use of POCUS.

\section{Discussion}

POCUS is a component of patient management in the ED that has been researched and developed since the 1990s. Focused cardiac ultrasound can be used to evaluate global function, pericardial effusion, and right ventricle dilatation [1]. In addition, the McConnell sign with a dilated right ventricle is identified as pulmonary thromboembolism by POCUS.

The McConnell sign is a significant regional wall motion contractility of the apex though akinesis or hypokinesis of the middle and base of the free wall of the right ventricle in systole $[2,3]$. It is interpreted as the effect of increased effort of the left ventricle at its apex requiring compensation for the abnormal size, function and pressure of the right ventricle. McConnell reported RV dysfunction with akinesia of the RV mid-free wall by comparing quantitative and qualitative segmental motion during acute pulmonary embolism, pulmonary hypertension, and in normal control cases. This technique is reported to have $77 \%$ sensitivity and $94 \%$ specificity for the diagnosis of acute pulmonary embolism, with a positive predictive value of $71 \%$ and a negative predictive value of $96 \%$ [4].

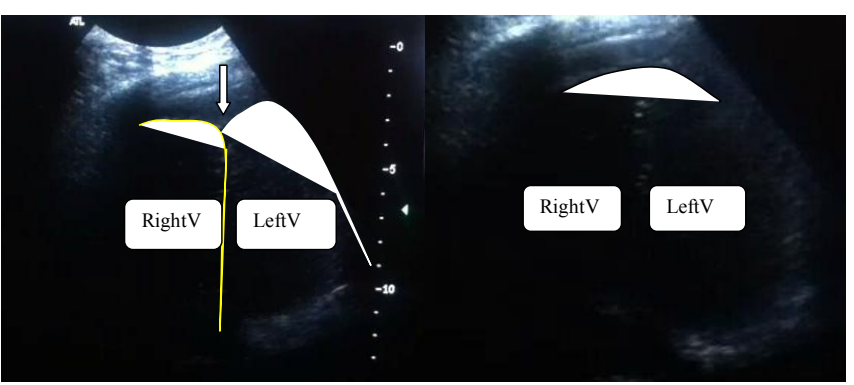

Fig. 1(A): Contraction of the apex of right ventricle on systole. (B): Diastole.
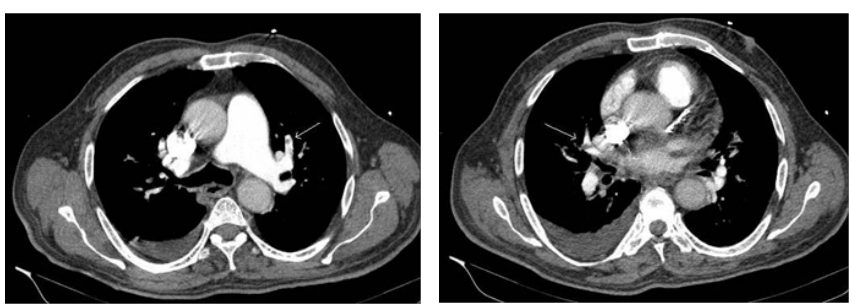

Fig. 2(A,B): Example of sections in computerized tomography angiography.

In another study, the McConnell sign had 96\% specificity and 16\% sensitivity when transthoracic echocardiography findings were analyzed within 48 hours of CT for pulmonary embolism [5]. There are several potential explanations for abnormal RV wall motion, including right ventricle infarct, and pulmonary hypertension [6]. However, abnormal RV free wall motion in pulmonary hypertension is distinct in that it affect all regions of the tissue [4]. Consequently, emergency medicine has to solve the acute clinical problems by providing the differential diagnosis and medical care in a short time. POCUS is the facilitator in this process. Even an old and large probe used for breast ultrasound prior in another clinic can solve the emergency question at point of care in a short time.

The McConnell sign with a dilated right ventricle on POCUS confirmed the bilateral thromboembolus on pulmonary branches. This was of significant help in the differential diagnosis, treatment, correct primary consultation, and hospitalization of this emergent and critical patient. 
Contributors: BG: Manuscript writing, case management; EK: manuscript revision, radiological investigations; EK will act as guarantor. Both authors approved final version of the manuscript.

Funding: None; Competing interests: None stated.

\section{References}

1. Via G, Hussain A, Wells M, Reardon R, ElBarbary $\mathrm{M}$, Noble VE, et al. International evidence-based recommendations for focused cardiac ultrasound. J Am Soc Echocardiogr. 2014;27:683.e1-683.e33.

2. Brenes-Salazar JA. McConnell's echocardiographic sign in acute pulmonary embolism: still a useful pearl. Heart Lung Vessel. 2015;7:86-88.
3. Haller EP, Nestler DM, Campbell RL, Bellamkonda VR. Point-of-care ultrasound findings of acute pulmonary embolism: McConnell sign in emergency medicine. J Emerg Med. 2014;47:19-21.

4. McConnell MV, Solomon SD, Rayan ME, Come PC, Goldhaber SZ, Lee RT. Regional right ventricular dysfunction detected by echocardiography in acute pulmonary embolism. Am J Cardiol. 1996;78:469-473.

5. Lodato JA, Ward RP, Lang RM. Echocardiographic predictors of pulmonary embolism in patients referred for helical CT. Echocardiography. 2008;25:584-590.

6. Platz E, Hassanein AH, Shah A, Goldhaber SZ, Solomon SD. Regional right ventricular strain pattern in patients with acute pulmonary embolism. Echocardiography. 2012;29: 464-470. 